\title{
Tissue preparation for simultaneous flow cytometric quantitation of tumour associated antigens and DNA in solid tumours
}

\author{
P A van Dam, J V Watson, D G Lowe, H Cox, M Curling, J H Shepherd
}

\begin{abstract}
A multiparameter flow cytometric assay for the simultaneous study of tumour associated antigens (TAA) and DNA in fresh solid tumours was devised. Cell suspensions were prepared by disaggregating unfixed solid tumour samples mechanically over a stainless steel mesh. Indirect immunofluorescence was used to identify the TAA, and DNA was stained with propidium iodide. Cell morphology was well preserved, cell clumping was negligible, and high quality indirect immunofluorescence and DNA staining were obtained.

The technique is simple, rapid, and reproducible. Multiparameter assays can be developed to study prognostic indicators such as membrane oncoproteins, receptors, and multidrug resistance in solid tumours. With a suitable panel of antibodies the technique might become an aid in the differential diagnosis and biochemical diagnosis of some solid tumours.
\end{abstract}

In theory flow cytometry can be used to define and measure any cell constituent, provided that a fluorescent tracer is available that reacts specifically and stochiometrically with that constituent. Quantitative multiparameter flow cytometric analysis of surface antigens and DNA has become a well established and reliable technique in the investigation of lymphoreticular cells. ${ }^{1}$ In solid tumours, however, flow cytometry has mainly been used for DNA analysis, and more recently for quantitative assay of nuclear antigens. ${ }^{1-7}$ There has been little work on flow cytometric determination of tumour associated antigens (TAA) in single cell suspensions of solid tumours because of the difficulties of tissue preparation and preservation of the antigenic determinants on the cell surface.

A multiparameter technique for the flow cytometric quantification of TAA expression and DNA analysis should provide high resolution of DNA content (low coefficient of variation of the DNA diploid peak), minimal cell clumping, well preserved immunofluorescence staining patterns and satisfactory morphology, as well as ease and speed of cell fixation and staining. ${ }^{1-7}$ In this paper we report a method of preparing cells for multiparameter analysis that meets these criteria and so could have many research and clinical applications.

\section{Methods}

Fresh tissue samples were obtained from normal and neoplastic tissues. Normal cervical and ovarian specimens were obtained from patients who had had hysterectomies for leiomyomas or dysfunctional uterine bleeding; normal lymph nodes were obtained from patients who had had surgery for stage $1 \mathrm{~b}$ cervical carcinoma. There were 10 primary carcinomas of the ovary (eight serous, one mucinous, and one endometrioid carcinoma); five squamous cell carcinomas of the cervix; three adenocarcinomas of the endometrium; two ovarian metastases from ductal carcinomas of the breast; two adenocarcinomas of the colon; two adenocarcinomas of the stomach; five normal ovaries; three normal cervices; one normal placenta; and five normal lymph nodes.

Samples were dissected free from fat and necrotic tissue and divided into two contiguous blocks. The first was fixed in methacarn (methanol:chloroform:acetic acid $60: 30: 10),{ }^{8}$ and processed as for routine histological specimens except that the initial formalin immersion stages were omitted. Sections were stained for TAA by the indirect immunoperoxidase technique.

The second block from the tissue was immediately disaggregated mechanically over a 16 gauge stainless steel mesh by mincing it into tiny fragments with scissors. Then a solution containing phosphate buffered saline (PBS: $8.0 \mathrm{~g} / 1 \mathrm{NaCl}, 1.15 \mathrm{~g} / 1 \mathrm{Na}_{2} \mathrm{HPO}_{4}, 0.20$ $\mathrm{g} / 1 \mathrm{KCl}, 0.10 \mathrm{~g} / 1 \mathrm{MgCl}_{2} 6 \mathrm{H}_{2} \mathrm{O}$ and $0.075 \mathrm{~g} / 1$ $\mathrm{CaCl}_{2} ; \mathrm{pH} 7 \cdot 4$ ) with $1 \%$ bovine serum albumin (BSA: Calbiochem, La Jolla, California, USA) at $4^{\circ} \mathrm{C}$ was gently flushed 30 to 50 times over the minced tissue to wash through the mesh as many cells as possible. The cells collected by this method were separated from remaining tissue fragments by passage through the mesh. The suspension was then centrifuged at $100 \times g$ for three minutes and the pellet resuspended in the buffer solution, with a final cell concentration of about $10^{6}$ cells $/ \mathrm{ml}$. The tissue fragments not passing through the mesh were fixed in methacarn and processed for indirect immunoperoxidase staining to assess the effectiveness of the dissociation method.

The effect of adding fetal calf serum and rabbit serum, which are known to contain compounds that inhibit proteolytic enzymes and stabilise cell membranes, was also studied at concentrations of $0,1,5,10$ and $20 \%$ in 10 different tissues. Normal rabbit serum in particular might also reduce the non-specific binding of the secondary rabbit anti-mouse 


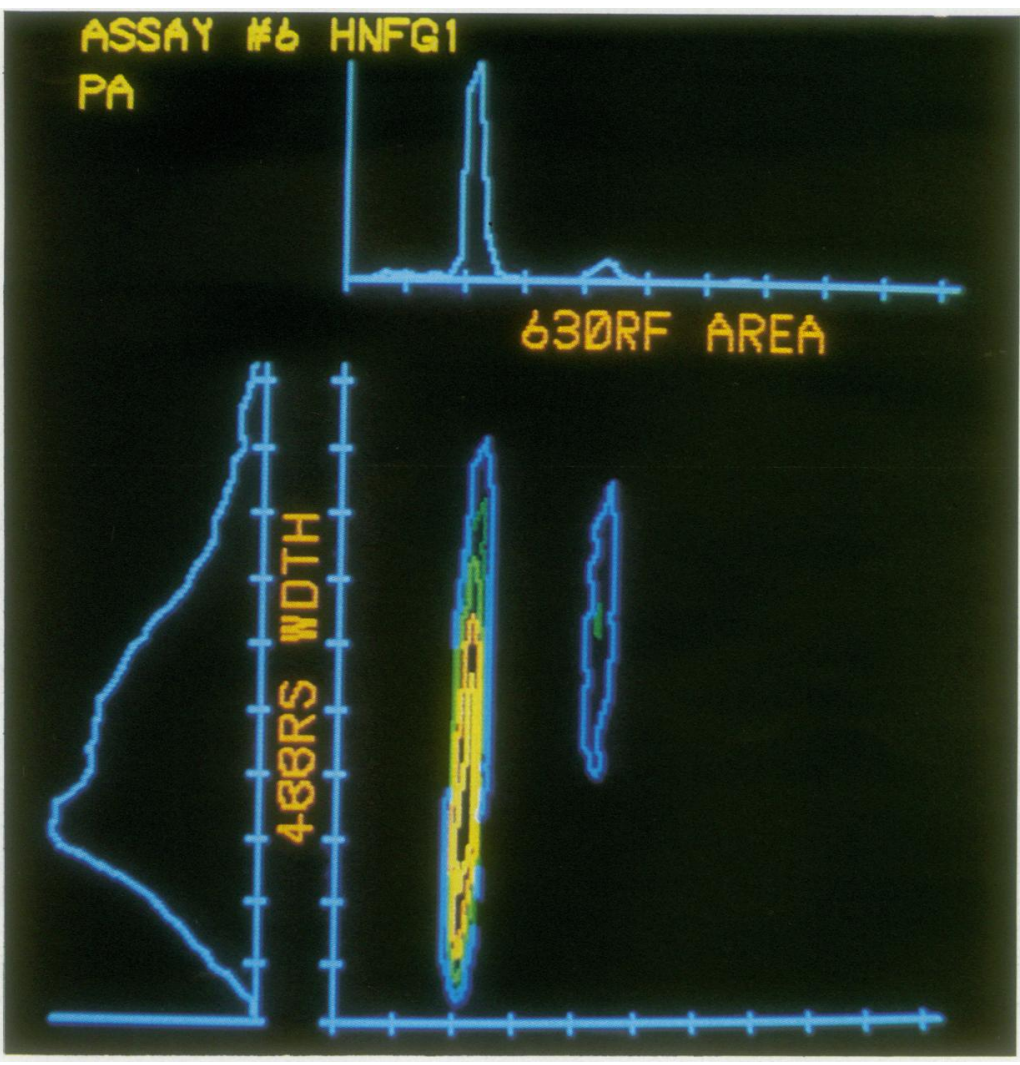

Figure 1 Ninety degree light scatter (pulse width) is plotted on the ordinate against $D N A$ on the abscissa for a DNA diploid serous cystadenocarcinoma. No gating has been done on these data. Contour lines are drawn around areas containing two, 10, 20, 40, 80, and 160 cells.

antibody. The amount of cell loss, reduction of the coefficient of variation of the DNA histograms, background fluorescence and TAA staining were measured.

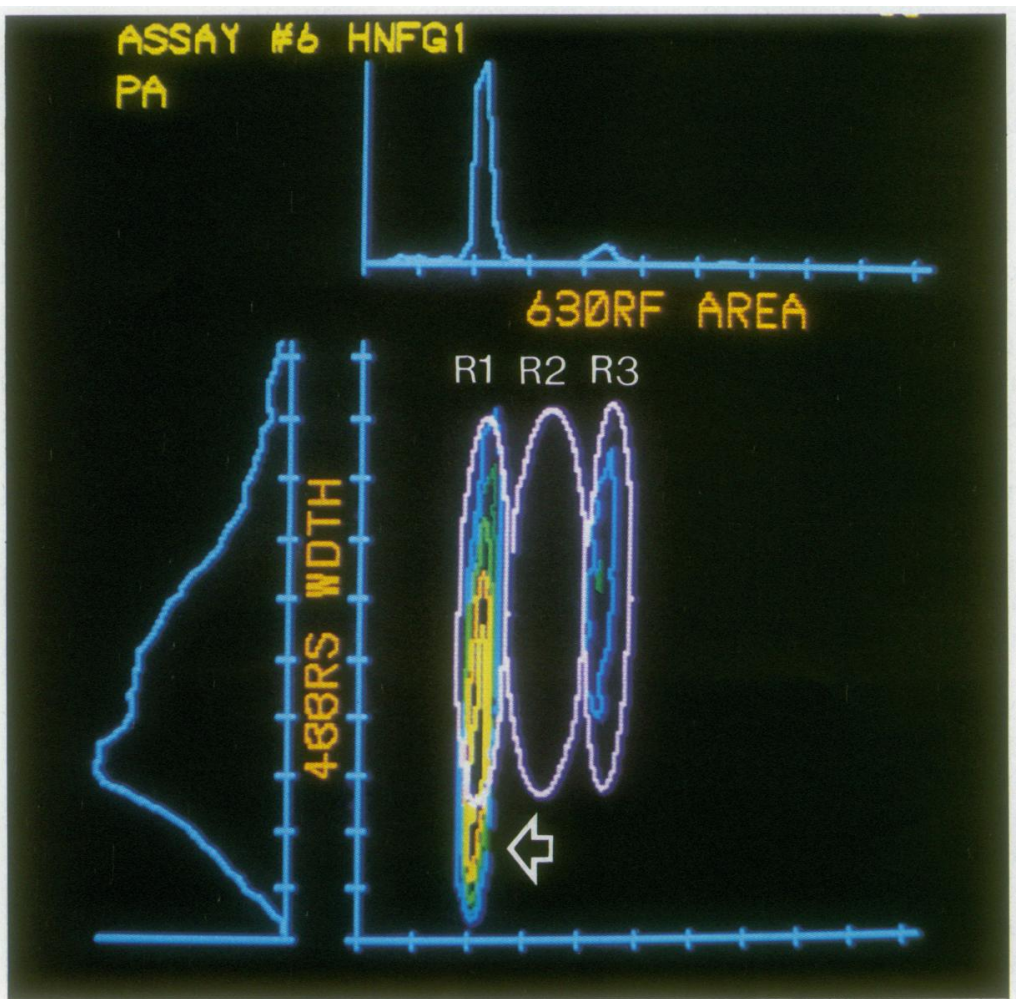

Figure 2 Three elliptical regions $R_{1}, R_{2}$, and $R_{3}$ were set over the G0/G1, $S$, and $G 2 / M$ compartments, respectively, of the same sample as in fig 1 . The debris, most low volume inflammatory and stromal cells (arrow), and cell clumps were not included in the gated regions.

\section{ANTIBODIES}

The development and characterisation of the monoclonal antibodies HMFG1, HMFG2, and SM3 have been described. ${ }^{910}$ Briefly, HMFG1 and HMGF2 antibodies attach to different tumour associated epitopes on a high molecular weight glycoprotein molecule, polymorphic epithelial mucin, found in human milk. SM3 antibody was raised against deglycosylated mucin ("stripped" mucin) purified by immunoaffinity chromatography from human skimmed milk and was chosen for the study because of its reactivity with partly and wholly stripped mucin and lack of reactivity with intact mucin. ${ }^{8}$ The antibodies were all in the IgG subclass and were kindly provided by the Imperial Cancer Research Fund at a concentration of $1 \mathrm{mg} / \mathrm{ml}$. Fluorescein isothiocyanate conjugated rabbit antimouse IgG (Dako Ltd, Denmark) was used to stain the primary antibody.

\section{STAINING OF TAA AND DNA}

All of the following staining procedures were performed on each of 10 different tissues. The suspension was filtered through a $35 \mu \mathrm{m}$ nylon mesh to remove large clumps. Part of this suspension was used for flow cytometry and the rest was used as a control for immunocytochemistry. One millilitre samples containing about $10^{6}$ cells were centrifuged. Two of the resulting pellets were resuspended in $30 \mu \mathrm{l}$ PBS with $1^{\circ}$ BSA and used as fluorescence controls. The others were resuspended in $30 \mu \mathrm{l}$ of the primary antibody (HMFG1, HMFG2, or SM3) at dilutions in PBS with $1^{\circ}, \mathrm{BSA}$ of 1 in 20,1 in 40,1 in 100,1 in 200,1 in 500,1 in 5000 and 1 in 50000 and incubated for one hour at room temperature to establish the optimal dilution of the primary antibody.

When the optimal dilution was found, the cell suspensions were incubated with this dilution of the antibody for $15,30,45,60,120$ and 180 minutes to determine the optimal incubation period at room temperature. The pellets were washed twice in PBS to remove free primary antibody. All of the pellets but one were then incubated with $30 \mu \mathrm{l}$ of dilutions of 1 in 20;1 in 40,1 in 100,1 in 200,1 in 500 and 1 in 5000 of fluorescein isothiocyanate (FITC) conjugated rabbit anti-mouse immunoglobulin (Dako, Denmark) for one hour. The remaining pellet was incubated with PBS alone. The unbound fluoresceinated antibody was removed by washing the cells twice with PBS after one hour.

To determine the optimal incubation period for the FITC conjugated secondary antibody, cells were incubated with the optimal concentration of it for $15,30,45,60,120$ and 180 minutes. The pellets were then resuspended in a solution of $30 \mu \mathrm{l}$ PBS containing ribonuclease (Sigma; $0.05 \mathrm{mg} / \mathrm{ml}$ ) and propidium iodide (Calbiochem Ltd; $0.05 \mathrm{mg} / \mathrm{ml}$ ) which counterstained the DNA red against the green staining of the TAA. One set of control cell suspensions was stained with propidium iodide alone and another with only propidium iodide and the second antibody (the fluores- 
Figure 3 Green plotted on the abscissa against the cell frequency on the ordinate for the region $R$, shown in fig 2 . There is a wide range of HMFG1 staining, reflecting heterogeneity within the tumour. fluorescence of HMFG1

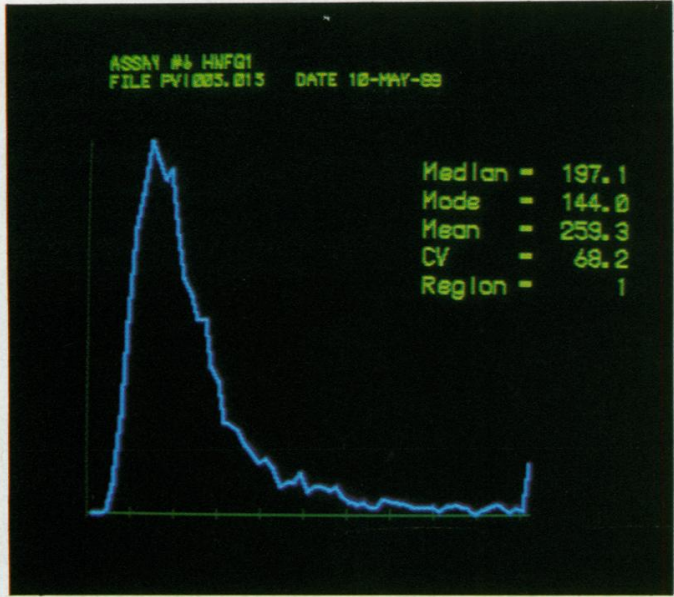

cence control), with the same dilutions and incubation times as those used for the TAA samples.

\section{FLOW CYTOMETRY}

The cells were analysed simultaneously for TAA expression and DNA in the Cambridge Medical Research Council custom built, dual laser flow cytometer, ${ }^{11}$ which incorporates a modified flow chamber to increase light collection efficiency. ${ }^{12}$ The Innova 70-5 argon ion laser (Coherent, Palo Alto, California) was tuned to $488 \mathrm{~nm}$ at a light power of $100 \mathrm{~mW}$, which excites red fluorescence from the propidium iodide/DNA complex and green fluorescence from the fluorescein labelled TAA. The green and red signals were separated by a $580 \mathrm{~nm}$ dichroic mirror (Zeiss Ltd, West Germany) and the respective photodetectors were guarded by a $515-560 \mathrm{~nm}$ band pass filter for green and a $630 \mathrm{~nm}$ long pass filter for red. Forward and $90^{\circ}$ light scatter signals were also collected. Calibration and setup of the instrument, data collection, and processing have been described previously. ${ }^{13}$

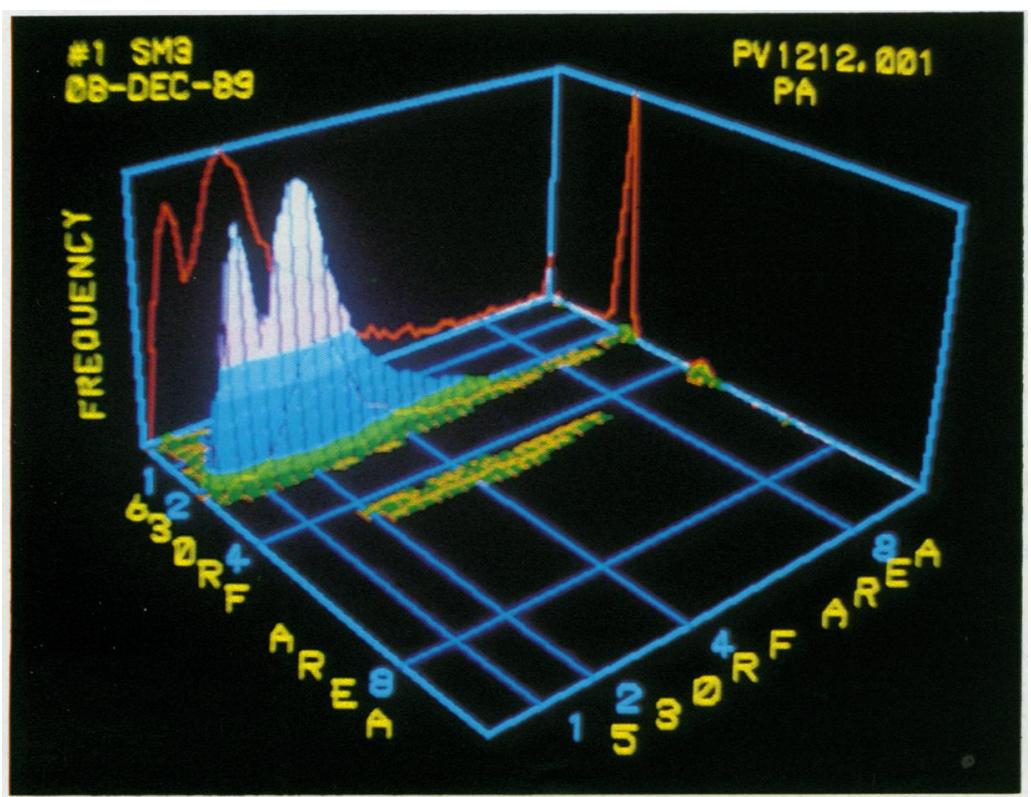

Figure 4 Three dimensional image of a preparation from an ovarian carcinoma, showing the green fluorescence ( $530 R F)$ of SM3 plotted on the $X$ axis, red fluorescence $(630 R F)$ of DNA on the $Z$ axis, and cell frequency on the $Y$ axis. The tumour is DNA diploid and shows typical heterogeneous SM3 expression.
Five parameters were assessed as a crosscorrelated data file ${ }^{14}:$ DNA, TAA fluorescence, forward scatter pulse width, $90^{\circ}$ scatter width, and $90^{\circ}$ scatter area.

\section{DATA ANALYSIS}

The $90^{\circ}$ scatter pulse width, which gives a measurement of cell diameter, was plotted against the results for DNA (fig 1). The values for cell debris, single nuclei, cell clumps, inflammatory cells and some of the stromal cells could therefore be identified and eliminated from subsequent measurements. Only particles that had values for both DNA and cell diameter were considered to be intact cells: cell debris such as enucleated or broken cells gave signals for diameter but not DNA, and bare nuclei gave signals for DNA but had only small signals for diameter. Intact inflammatory cells had a normal DNA index and diameters at the lower end of the scale.

DNA diploid peaks could partly be the result of stromal and inflammatory cells, which would make interpretation of results in DNA diploid tumours more difficult. The cytological controls showed that stromal cells were generally smaller than tumour cells. A large percentage of the stromal and inflammatory cells could be gated out successfully by excluding the small volume diploid cells. This was confirmed in separate experiments, in which cell suspensions from fresh and cryopreserved ovarian and cervical neoplasms and normal ovary were incubated with dilutions of leukocyte common antigen (LCA; Dako, Denmark), and suspensions from cryopreserved normal ovary and endometrium with the low molecular weight cytokeratin marker CAM 5.2 (Imperial Cancer Research Fund). The region of small volume DNA diploid cells contained most of the cells staining positive with LCA $(76-94 \%$, median $85^{\circ}{ }_{0}$ ) and was negative for TAA and CAM 5.2. The epithelial cells in the endometrial samples stained with CAM $5 \cdot 2$ and were included in the region that contained the cells positive for TAA. Even so, it was not possible entirely to resolve the cells in this region in DNA diploid tumours into "normal" and "tumour" cell components.

Elliptical regions were set on the entire intact cell population and on the $\mathrm{G} 0 / \mathrm{Gl}, \mathrm{S}$, and G2/M populations of the DNA diploid tissues (fig 2). In DNA aneuploid tumours the G0/G1 diploid and DNA aneuploid cell populations were defined. The five parameters listed above were measured for each of the gated regions. By subtracting the median green fluorescence in the fluorescence control sample $\left(F_{b}\right)$ from that in the TAA sample $\left(F_{t}\right)$, the TAA fluorescence could be calculated for each subset of cells (fig 3) and for the complete cell population (figs 4 and 5). As most experiments were comparisons of staining procedures the TAA specific fluorescence $\left(F_{s}\right)$ was defined as $\left(F_{t}-F_{b}\right) / F_{t} \times 100 .{ }^{15}$ The optimal staining procedure was taken as the procedure that gave the most intense fluorescence with the least amount of non-specific staining (highest $F_{s}$ ). The reproducibility of the technique was assessed by generating three histograms (each based 
Figure 5 Intensity of green fluorescence plotted on the abscissa against the number of cells in the

fluorescence control sample $\left(F_{b}\right)$ and in the sample stained with $H M F G 1$

$\left(F_{t}\right)$ in a serous cystadenocarcinoma. The tumour cells stained strongly with HMFG1.

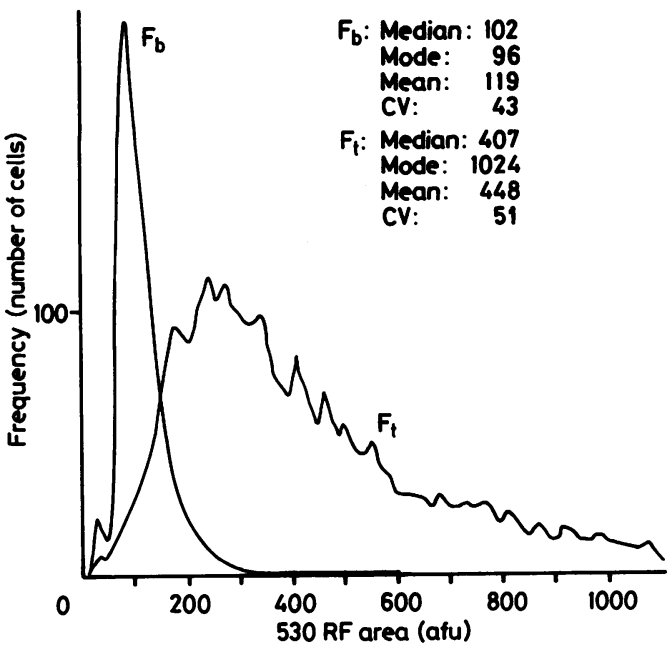

on measurement of $10^{4}$ cells) for each sample and by simultaneously preparing the same tissue sample three times in the same way. As the distribution values for TAA expression were skewed, non-parametric tests were used to analyse the data. Statistical differences are given by the $p$ values indicated in the text, and a difference of $p<0.05$ was regarded as significant.

Specificity controls for antibody binding were performed with blocking assays. AntiSM3 binding was blocked by its immunogen, the SM3 peptide. Two monoclonal antibodies raised against nuclear oncoproteins, CD5 and M14 (Cambridge Research Biochemicals, Harston, England) which do not recognise mucin TAA or membrane structures gave no signal above background in the tissues analysed.

From the cell suspensions used for the flow cytometric quantitation of TAA a cytology smear was made. This was fixed in methanol and air dried. In addition, $3 \mu \mathrm{m}$ sections were cut from the paraffin wax embedded tissue blocks that were taken from the fresh tissues for immunohistochemistry. The indirect immunoperoxidase technique was used to stain the smears and sections for TAA. To assess TAA expression in the smears and sections 200-400 intact epithelial cells were examined without knowledge of the result of the flow cytometric analysis. The extent of staining was scored as follows: $-0-5 \%,+6-25 \%,++26-$ $50^{\circ}$, and $+++51-100 \%$ of epithelial cells staining positively for TAA.

\section{Results}

Mechanical dissociation of the tissue samples was satisfactory in all cases. The average total cell yield per gram of tissue for these specimens was $4.8 \times 10^{7}$ cells $/ g$ (standard deviation $1.9 \times 10^{7}$ cells $/ g$ ). The cell yield was significantly higher in neoplastic tissues $(5 \cdot 7$ $(1.6) \times 10^{7}$ cells $/ g$ ) than in the normal tissues studied $\left(1.2(1.4) \times 10^{7}\right.$ cells $/ g$ ) (ANOVA $\mathrm{p}=0.005)$. Histological examination of the residual tissue fragments in malignant tumours showed large amounts of stroma with areas of mainly intact tumour cells. The cytospin slides prepared from the suspensions that were subsequently used for flow cytometry showed that

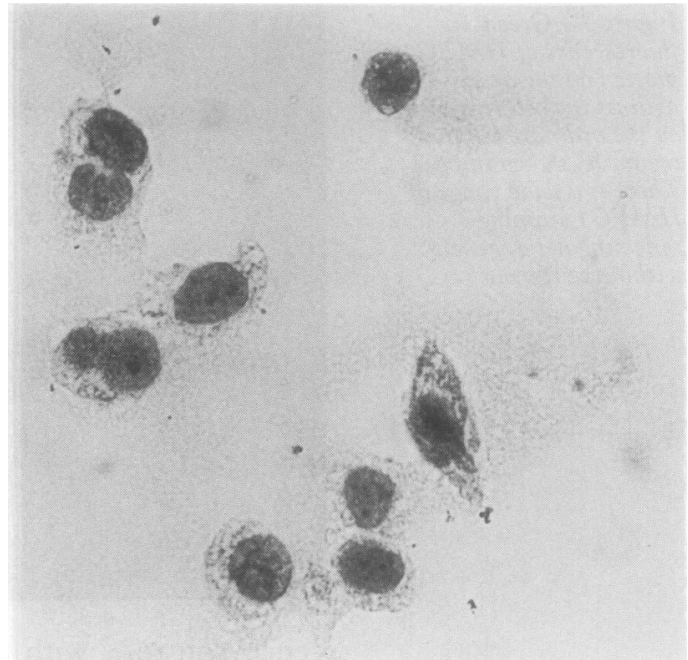

Figure 6 Cytology preparation of a cell suspension from the tumour in fig 4, showing large polyhedral tumour cells.

cellular morphology was well preserved (fig 6); in the suspensions prepared from malignant tumours most of the cells were tumour cells. These cells were in the larger volume range and showed heterogeneous TAA staining. The stromal cells in most cases were much smaller and showed hardly any TAA expression.

The quality of the DNA staining, assessed by the coefficient of variation of the DNA diploid G0/G1 peak, was good. In normal ovaries and normal lymph nodes the coefficient of variation lay between $2 \cdot 5$ and $6 \cdot 1 \%$ (mean $4 \cdot 7 \%$ ). DNA aneuploid cell populations were shown in 17 of the 24 malignant specimens. The amount of cell debris and clumping was very small (figs 1 , $2,4)$.

A major technical obstacle to the

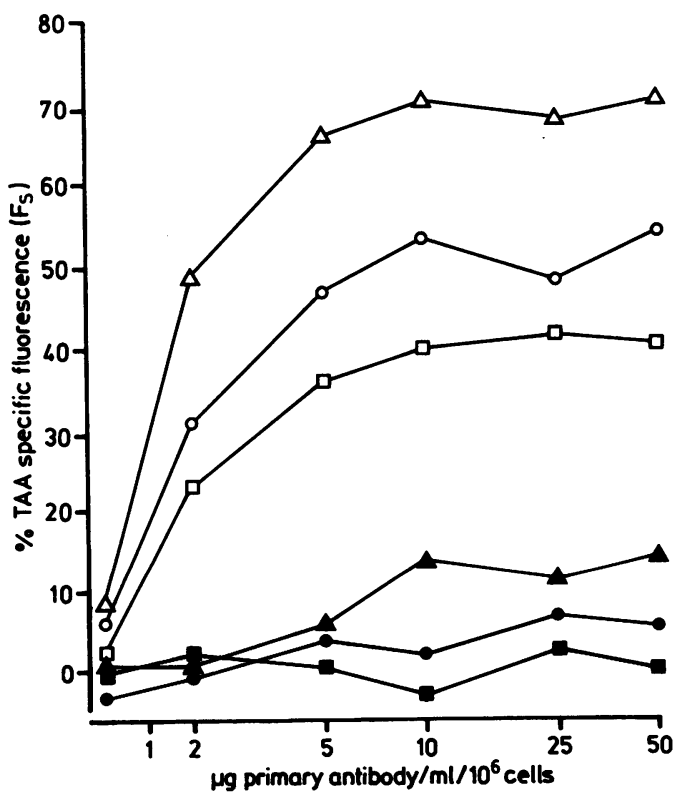

Figure 7 Titration of SM3 (squares), HMFG1 (triangles), and HMFG2 (circles) in a serous cystadenocarcinoma of ovary (open symbols) and normal ovary (closed symbols). The fluorescence curve for SM3 in normal ovary was relatively flat, showing that nonspecific binding of primary antibody did not contribute to $F_{b}$ to any great extent. The results were similar in all of the 10 cases studied. 
Figure 8 Titration of FITC labelled rabbit antimouse secondary antibody in a serous

cystadenocarcinoma of ovary (open symbols) and normal ovary (closed symbols). Cells were stained with $30 \mu \mathrm{l}$ of $S M 3$ (squares), $H M F G 1$ (triangles), and HMFG2 (circles) per millilitre containing $10^{6}$ cells. The dilution of the secondary antibody was varied. Optimal fluorescence was obtained with a 1 in 100 dilution of the secondary antibody. The results were similar in all of the cases studied.

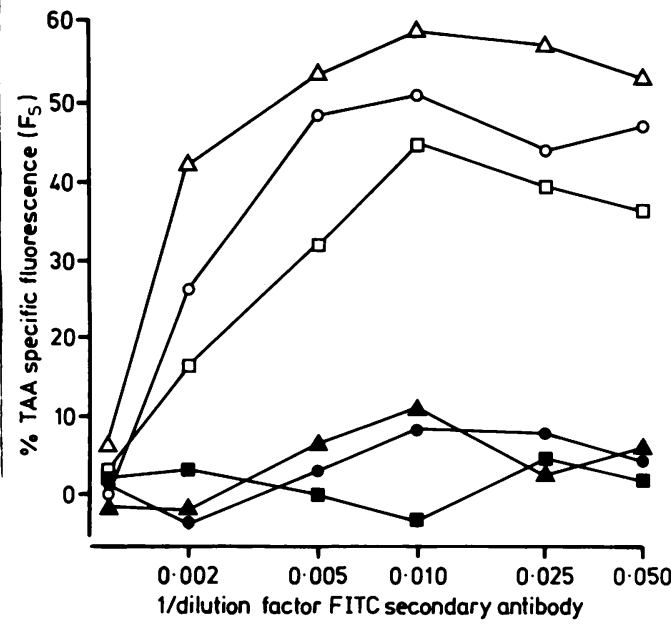

measurement of TAA expression by immunofluorescence is background fluorescence $\left(F_{b}\right)$. This includes non-specific binding of primary and secondary antibody, spectral overlap of propidium iodide fluorescence, and autofluorescence. Background fluorescence was found to be significantly lower in normal tissues (median 132 arbitrary fluorescence units, lower quartile 108, upper quartile 176) than in malignant tumours (median 183, lower quartile 127, upper quartile 256) ( $\mathrm{p}=0.008$ Mann Whitney $U$ test). Propidium iodide spectral overlap and autofluorescence, assessed by comparing cell suspensions that were stained for DNA with and without TAA staining, comprised $23-58^{\circ}$ o (average $34^{\circ}$ ) of $\mathrm{F}_{\mathrm{b}}$. The remaining $F_{b}$ was therefore presumably due to non-specific binding and trapping of the primary and secondary antibody. A reduction of the $F_{b}: F_{t}$ ratio was obtained by adjusting the method of preparation. This involved optimising the dilutions of the primary and secondary antibodies, optimising the ratio of the reaction volume and the number of cells per sample, choosing the best staining time and increasing the number of washing steps.

The saturating antibody dilutions and patterns of antibody staining for SM3, HMFG1, and HMFG2 were found to be in the same range. The checkerboard titration of SM3, HMFG1, and HMFG2 illustrated two points: excess of primary antibody was achieved with a 1 in 100 dilution $(10 \mu \mathrm{g}$ antibody $/ \mathrm{ml}$ containing

SM3, HMFG1, and HMFG2 expression in neoplastic and non-neoplastic DNA diploid tissues in the $G 0 / G 1, S$, and $G 2 / M$ phases of the cell cycle $(n=21)$

\begin{tabular}{|c|c|c|c|}
\hline$T A A$ & $G 0 / G 1^{\star}$ & $S^{\star}$ & $G 2 / M^{\star}$ \\
\hline $\begin{array}{l}\text { Without co } \\
\text { SM3 } \\
\text { HMFG1 } \\
\text { HMFG2 }\end{array}$ & $\begin{array}{l}r \text { cell surface } \\
118(25-192) \\
223(79-397) \\
160(46-214)\end{array}$ & $\begin{array}{l}140(67-211) \\
278(144-454) \\
217(90-253)\end{array}$ & $\begin{array}{l}171(88-274) \\
296(175-463) \\
242(152-287)\end{array}$ \\
\hline $\begin{array}{l}\text { With corre } \\
\text { SM3 } \\
\text { HMFG1 } \\
\text { HMFG } 2\end{array}$ & $\begin{array}{l}\text { ll surface } \\
5.34(1 \cdot 18-9.91) \\
10.53(3.98-19 \cdot 17) \\
8.08(2 \cdot 08-11.57)\end{array}$ & $\begin{array}{l}5.38(2 \cdot 38-7 \cdot 80) \\
9 \cdot 78(4 \cdot 45-16 \cdot 78) \\
7 \cdot 44(3 \cdot 29-8 \cdot 36)\end{array}$ & $\begin{array}{l}5 \cdot 45(2 \cdot 70-9 \cdot 06) \\
9 \cdot 57(5 \cdot 06-14 \cdot 55) \\
7 \cdot 19(4 \cdot 36-9 \cdot 15)\end{array}$ \\
\hline
\end{tabular}

* Median (lower quartile-upper quartile)

Significance of differences (Wilcoxon), for SM3, HMFG1, and HMFG2, respectively:

Without correction for cell size

G0/G1:S p $=0.013,0.011,0.002$.

G0/G1:G2/M p $=<0.001,0.001,<0.001$

$\mathrm{S}: \mathrm{G} 2 / \mathrm{M} \quad \mathrm{p}=<0.001,0.231,0.071$

With correction for cell size

None of the differences was significant.
$10^{6}$ cells) without loss in $\mathrm{F}_{\mathrm{s}}$; and as the TAA specific fluorescence curve for a negative normal sample was flat, trapped or nonspecifically bound primary antibody hardly contributed to $F_{b}$ (fig 7). This shows that samples can be incubated with only the FITC labelled second antibody and propidium iodide to correct for $F_{b}$. Increasing the purity and dilution of the secondary antibody should result in an increase in $F_{s}$, as greater dilutions of antibody under saturation conditions permit less non-specific binding. The data in fig 8 suggest that this is so, and that the secondary antibody should optimally be used in a 1 in 100 dilution.

The optimal incubation time for the primary and secondary antibody was found to be 60 minutes. After two washes the effect of further washing to remove the trapped antibody was negligible in all cases. With the optimised preparative technique $F_{s}$ up to $75 \%$ could be achieved. Intra-assay and interassay reproducibility were $3.5 \%$ and $16 \%$, respectively.

To try to reduce $F_{b}$ and improve reproducibility normal rabbit serum was added to all reagents in the reaction. This resulted in no significant reduction of $F_{b}$ or increase of $F_{s}$, but gave slightly less cell loss. The best concentrations were $5-10^{\circ}$ rabbit serum in PBS; there was no appreciable difference between these concentrations. Similar results were obtained by adding $5-10 \%$ fetal calf serum to the reagents.

Expression of SM3, HMFG1, and HMFG2 was absent or very low in the normal tissues (24, lower quartile 8 , upper quartile $33.5 ; 67$, lower quartile $37 \cdot 2$, upper quartile 89.5 ; and 45 , lower quartile 22 , upper quartile 58 , respectively) and significantly higher in the malignant tumours $(258$, lower quartile 184 , upper quartile $335 ; 508$, lower quartile 257 , upper quartile 818 ; and 371 , lower quartile 258 , upper quartile 497 , respectively) $(\mathrm{p}<0.001, \mathrm{p}<0.001$ and $\mathrm{p}<0.001$, respectively, Mann Whitney $U$ test). A wide variation in TAA expression from negative to strongly positive cells was a common finding in cells with similar DNA content.

In DNA diploid tissues the total TAA expression was highest in $\mathrm{G} 2 / \mathrm{M}$ phase cells, intermediate in $S$ phase cells, and lowest in G0/ G1 phase cells (table). From measurements of the $90^{\circ}$ scatter pulse width, however, it was apparent that the cells in $\mathrm{G} 2 / \mathrm{M}$ phase were larger than those in $S$ phase which were in turn larger than those in $\mathrm{G} 0 / \mathrm{Gl}$ phase. As our computer software does not allow for size correction on a cell by cell base it is not easy to account for. Assuming that cells can be regarded as spheres and that TAA are located mainly at the cell membrane (thus neglecting cytoplasmatic TAA expression) and as the green fluorescence and $90^{\circ}$ pulse scatter width were measured in arbitrary units, $\mathrm{F}_{\mathrm{t}}-\mathrm{F}_{\mathrm{b}}$ fluorescence of the gated $\mathrm{G} 0 / \mathrm{G} 1, \mathrm{~S}$, and $\mathrm{G} 2 / \mathrm{M}$ phase cells was divided by the square of their respective median $90^{\circ}$ pulse scatter width signal. Thus we found no significant changes of TAA expression during the cell cycle (table).

In DNA aneuploid tumours no difference 
Figure 9 Extent of staining with $S M 3$

(squares), HMFG1 (triangles), and $H M F G 2$ (circles) scored subjectively on histological sections plotted against staining with the same antibodies measured by flow cytometry.

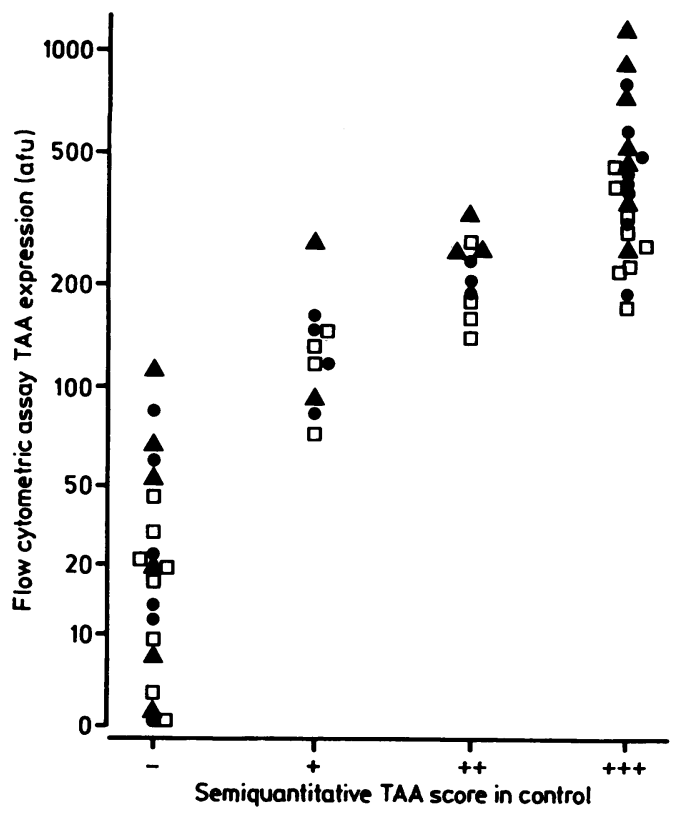

was found between TAA expression in the DNA aneuploid and DNA diploid G0/G1 compartments. There was good correlation between the flow cytometry and the immunocytological and immunohistochemical controls (fig 9).

\section{Discussion}

A few papers have been published over the past few years describing methods for flow cytometric quantitative analysis of TAA expression in cultured cells and in cell suspensions of disaggregated solid tumours. ${ }^{16-27}$ The preparative methods used in these studies were often rather empirical and time consuming and did not take into account important variables such as method of cellular fixation and length of storage. ${ }^{20-26}$ Some workers used single parameter flow cytometric assays for the quantitation of TAA expression, ${ }^{162}$ which, compared with multiparameter assays, do not permit as good a correction for the fluorescence from cellular debris and clumps. ${ }^{28}$

Our investigation was therefore undertaken to try to establish a baseline for the development of a rational and straightforward multiparameter flow cytometry technique for the assay of TAA expression in fresh, solid tumours. In particular we wanted to see whether this method was practical, quantitative, and reproducible. The influence of cellular fixation, preservation, and storage on multiparameter flow cytometric TAA measurements are being studied.

The method described here may need to be adjusted for multiparameter flow cytometric assay of TAA in single cell suspensions from different solid tumours and for different TAA. We used mechanical dissociation on the tissue samples as it has proved to be better than enzymatic dissociation for breast, colonic, and ovarian tumours ${ }^{29}{ }^{30}$ and it eliminates the risk of enzyme damage to the epitope of the membrane TAA. In other tumours, however, particularly those containing more fibrous tissue, enzymatic dissociation methods can produce higher quality single cell suspensions..$^{30-32}$ Our cytological controls showed a high percentage of intact tumour cells, and the DNA histograms showed that the preparations were of good quality with very small amounts of DNA debris. Histological examination of the minced tissue samples suggested that tumour cells had been taken into suspension more readily than stromal cells. Mechanical tissue dissociation therefore seemed to be quite suitable. In theory it is not possible to exclude selective destruction of a vulnerable population of cells with any of the available mechanical or enzymatic dissociation techniques, but from the small amount of debris in the cell suspensions we prepared mechanically this seems unlikely.

As we could assay SM3, HMFG1, and HMFG2 under saturated antibody conditions and could correct for background fluorescence, the method described is properly quantitative. If this method were to be used for the assay of other TAA a similar checkerboard analysis would have to be performed as the primary antibody might have a different purity and affinity and there might be a lower amount of epitope available.

Immunofluorescence analysis by flow cytometry with monoclonal antibodies is a valuable means of quantifying proteins in heterogeneous cell systems such as solid tumours. The capacity of flow cytometry to make measurements on a cell to cell basis makes it particularly suitable for assaying minority cell populations in a tumour. It has the advantage over other methods such as radioimmunoassay and immunoblot techniques that morphologically intact cells can be identified and separated from cell debris, clumps, and most inflammatory cells and stromal cells. Flow cytometry also enables direct correlations to be made between specific TAA concentrations and other variables such as DNA content. As flow cytometry does not give morphological information, however, it should always be used in combination with histology. Immunohistochemical controls are essential for the correct interpretation of the results of multiparameter flow cytometry assay and are especially so in tissues that have a high stromal cell to epithelial cell ratio, such as the ovary, where the impact of TAA positive epithelial cells can be diluted by the presence of large numbers of negative stromal cells in the sample.

Multiparameter assays can be developed to study prognostic indicators such as membrane oncoproteins, receptors, and multidrug resistance in solid tumours. The technique has obvious clinical applications in the management of immunotherapy and immunoimaging, and perhaps in monitoring intraperitoneal chemotherapy and lymphokine treatment. With a suitable panel of monoclonal antibodies, it might become an aid in the differential diagnosis and "biochemical" diagnosis of some solid tumours.

We thank Mr C N Hudson, Mr N Wathen, Mr M Setchell, Mr $M$ Gillard, and Mr G Evans for providing operative specimens, and Professor T Chard and Mr C N Hudson for their comments during the preparation of the manuscript. 
This study was supported by a European Community Cancer Research Grant and a NATO Research Fellowship.

1 Coon JS, Landay AL, Weinstein RS. Biology of disease: advances in flow cytometry for diagnostic pathology. Lab Invest 1987;57:453-79.

2 Watson JV, Sikora K, Evan GI. A simultaneous flow cytometric assay for c-myc oncoprotein and DNA in nuclei from paraffin embedded material. J Immunol Methods 1985;83:179-92.

3 Clevenger CV, Bauer KD, Epstein AL. A method for simultaneous nuclear immunofluorescence and DNA content quantitation using monoclonal antibodies and flow cytometry. Cytometry 1985;6:208-14.

4 Bauer KD, Clevenger CV, Endow RK, Murad T, Epstein AL, Scarpelli DG. Simultaneous nuclear antigen and DNA content quantitation using paraffin-embedded colonic tissue and multiparameter flow cytometry. Cancer Res 1986;46:2428-34

5 Hendy-Ibbs P, Cox H, Evan GI, Watson JV. Flow cytometric quantitation of DNA and c-myconcoprotein in archival biopsies of uterine cervix neoplasia. $\mathrm{Br} \mathrm{J}$ Cancer 1987;55:275-82.

6 Sikora K, Chan S, Evan G, et al. C-myc oncogene expression in colorectal cancer. Cancer 1987;59:1289-95.

7 Lincoln ST, Bauer KD. Limitations in the measurement of c-myc oncoprotein and other nuclear antigens by flow C-myc oncoprotein and other nuclear
cytometry. Cytometry 1989;10:456-62.

8 Girling A, Bartkova J, Burchell J, Gendler S, Gillett C Taylor-Papadimitriou J. A core protein epitope of the polymorphic epithelial mucin detected by the monoclonal antibody SM3 is selectively exposed in a range of primary carcinomas. Int J Cancer 1989;43:1072-6.

9 Taylor-Papadimitriou J, Peterson JA, Arklie J, Burchell J, Ceriani RL, Bodmer WF. Monoclonal antibodies to epithelium-specific components of the human milk fat globule membrane: production and reaction with cells in culture. Int J Cancer 1981;28:17-21.

10 Burchell J, Gendler S, Taylor-Papadimitriou J, Girling A, Lewis A, Millis R. Development and characterisation of breast cancer reactive monoclonal antibodies directed to breast cancer reactive monoclonal antibodies directed to the core protein

11 Watson JV. Dual laser beam focusing for flow cytometry through a single crossed cylindrical lens pair. Cytometry 1981;2:14-9.

12 Watson JV. A method for improving light collection by $600^{\prime \prime}$ "from square cross section flow cytometry chambers. Br J Cancer 1985;51:433-5.

13 Watson JV, Curling OM, Munn CF, Hudson CN Oncogene expression in ovarian cancer: a pilot study of c-myc oncoprotein in serous papillary ovarian cancer. Gynecol Oncol 1987;28:137-50.

14 Watson JV, Horsnell TS, Smith PJ. Data compression: 8 dimensional flow cytometric data processing with $28 \mathrm{~K}$ addressable computer memory. J Immunol Methods 1988;113:205-14.

15 Jacobberger JW, Fogleman D, Lehman JM. Analysis of intracellular antigens by flow cytometry. Cytometry 1986;7:356-64

16 Inoue S, Masami I, Shigetaka M, et al. Simultaneous flow cytometric detection of nuclear DNA and tumour associated antigens in lung cancers. Analyt Quant Cytol Histol 1988;10:243-50.

17 Sasaki K, Murakami T, Kawasaki S, Okita K, Takemoto T, Takahashi M. Change of $\alpha$-fetoprotein content during cell cycle of human hepatoma cells in vitro: flow cytometric analysis. Tumour Biology 1985;6:483-9.

18 Czerniak B, Darzynkiewicz Z, Staiano-Coico L, Herz F, Koss L. Expression of Ca antigen in relation to cell cycle in cultured human tumour cells. Cancer Res 1984;44:4342-6.

19 Marder P, Apelgren LD, Bumol TF. Comparative analysis of monoclonal antibody-drug conjugate binding by flow cytometry. J Immunol Methods 1987;96:165-70.

20 Valet G, Russmann L. Automated flow cytometric identification of colorectal tumour cells by simultaneous DNA, CEA antibody and cell volume measurements. $J$ Clin Chem Clin Biochem 1984;22:935-42.

21 Valet G, Ormerod MG, Warnecke HH, Benker G, Ruhenstroth-Bauer G. Sensitive three parameter flow cytometric detection of abnormal cells in human cervical cancer: a pilot study. J Cancer Res Clin Oncol 1981; 102:177-84.

22 Orntoft TF, Petersen SE, Wolf H. Dual parameter flow cytometry in transitional cell carcinomas: quantitation of DNA content and binding of carbohydrate ligands in cellular subpopulations. Cancer 1988;61:963-70.

23 Fowler WC, Maddock MB, Moore DH, Haskill S. Significance of multiparameter flow cytometric analysis of nificance of multiparameter flow cytometric analysis of

24 Sasaki K, Nagai M, Kato H, Torigoe T, Nagamine Y, Takahashi M. Flow cytometric analysis of tumour antigen TA-4 in cervical squamous cells. Gann 1984;75:703-6.

25 Paratto NP, Kimura AK. Isolation and visualisation of Met72-positive, metastatic variants present in $\mathrm{B} 16$ melanoma tumour masses. $J$ Cell Biochem 1988;36:311-22.

26 Czerniak B, Koss LG. Expression of Ca antigen on human urinary bladder tumours. Cancer 1985;55:2380-3.

27 Nozawa S, Sakyori M, Kunihiko Y, et al. A monoclonal antibody (MSN-1) against a newly established uterine endometrial cancer cell line (SNG-11) and its application to immunohistochemistry and flow cytometry. Am J Obstet Gynceol 1989;161:1079-86.

28 van Dam PA, Watson JV, Lowe DG, Shepherd JH. Flow cytometric quantitation of tumor associated antigens in solid tumors. Am J Obstet Gynecol 1990;163:698-9.

29 Ensley JF, Maciorowski Z, Pietraszkiewicz H, Hassan M, Crissman J, Valdivieso $M$. Selective and consistent aneuploidal cellular subpopulation losses in enzymatically versus mechanically dissociated human colon specimens analysed by flow cytometry. Proc Am Assoc Cancer Res 1987;28:248-51.

30 Chassevent A, Daver A, Bertrand G, et al. Comparative flow DNA analysis of different cell suspensions in breast DNA analysis of different cell suspe
carcinoma. Cytometry 1984;5:263-7.

31 Ensley JF, Maciorowski Z, Pietraszkiewicz H, et al. Solid tumour preparation for flow cytometry using a standard murine model. Cytometry 1987;8:488-93.

32 Slocum H, Pavelic Z, Rostum Y, et al. Characterisation of cells obtained by mechanical and enzymatic means from human melanoma, sarcoma and lung tumour. Cancer Res $1983 ; 41: 1428-34$. 